\title{
Impact of High-Flow Nasal Cannula Use on Neonatal Respiratory Support Patterns and Length of Stay
}

\author{
Suma B Hoffman MD, Natalie Terrell NNP, Colleen Hughes Driscoll MD, and \\ Natalie L Davis MD MMSc
}

\begin{abstract}
BACKGROUND: Heated humidified high-flow nasal cannula (HFNC) is thought to be comparable with nasal CPAP. The effect of multimodality mid-level respiratory support use in the neonatal ICU is unknown. The objective of this work was to evaluate the effect of introducing HFNC on length of respiratory support and stay. METHODS: A chart review was conducted on subjects at 24-32 weeks gestation requiring mid-level support (HFNC/nasal CPAP) 1 y before and after HFNC implementation. The 2 groups were compared for clinical and demographic data using $t$ test or chi-square analysis. Further, multivariate linear and logistic regression was done to determine significant risk factors for outcomes controlling for covariates. RESULTS: Eighty subjects were eligible in the pre-HFNC group, and 83 were eligible in the post-HFNC group. Subjects were similar in their baseline characteristics. In clinical outcomes, the post-HFNC group had higher rates of retinopathy of prematurity $(P=.02)$ and a trend toward higher bronchopulmonary dysplasia rates $(P=.063)$. The post-HFNC subjects had longer duration of mid-level support and were older at the time they were weaned to stable low-flow nasal cannula $(P<.05)$. Although the length of respiratory support and stay and corrected gestational age at discharge were similar, those in the preHFNC period were more likely to be receiving full oral feeds and be discharged home versus being transferred to an intermediate care facility $(P<.05)$. CONCLUSIONS: HFNC introduction was significantly associated with a longer duration of mid-level respiratory support, decrease in oral feeding at discharge, increased retinopathy of prematurity rates, and higher use of intermediate care facilities, leading us to examine our noninvasive ventilation and weaning strategies. Key words: neonate; high-flow nasal cannula; nasal CPAP; respiratory distress syndrome. [Respir Care 2016;61(10):1299-1304. (C) 2016 Daedalus Enterprises]
\end{abstract}

\section{Introduction}

Premature infants, especially those born at very low birthweight $(<1.5 \mathrm{~kg})$ and extremely low birthweight $(<1$

\footnotetext{
Drs Hoffman, Driscoll, and Davis are affiliated with the University of Maryland School of Medicine, Department of Pediatrics, Baltimore Maryland. Ms Terrell is affiliated with the University of Maryland Medical Center, Baltimore Maryland.

The authors have disclosed no conflicts of interest.

Dr Hoffman, Ms Terrell, Dr Driscoll, and Dr Davis presented a version of this work at the Eastern Society for Pediatric Research Annual Meeting, held March 21, 2015, in Philadelphia, Pennsylvania. Dr Hoffman, Ms Terrell, Dr Driscoll, and Dr Davis presented a version of this work as a poster at the Pediatric Academic Societies Annual Meeting, held April 25, 2015, in San Diego, California.
}

$\mathrm{kg}$ ), often have long lengths of stay in the neonatal ICU, presenting a heavy health-care resource burden. ${ }^{1}$ In this patient population, bronchopulmonary dysplasia (BPD) is a major cause of morbidity and mortality and contributes to the long stay in the neonatal ICU. ${ }^{2}$ It has been shown that the use of nasal CPAP reduces the length of oxygen dependence and hospital stay. ${ }^{3-6}$

However, nasal CPAP is not without drawbacks. This modality is nursing-intensive, can cause nasal breakdown, and can be poorly tolerated by patients. ${ }^{7,8}$ Due to these

\footnotetext{
Correspondence: Suma B Hoffman MD, Department of Pediatrics, University of Maryland, 110 South Paca Street, 8-S-163, Baltimore MD 21201. E-mail: shoffman@peds.umaryland.edu.
}

DOI: $10.4187 /$ respcare. 04668 
disadvantages, the use of heated humidified high-flow nasal cannula (HFNC) is increasing across the world as a noninvasive respiratory support alternative in neonates. ${ }^{9,10}$ This modality differs from nasal CPAP in that it is a flowbased support versus a pressure-based support. The heated and humidified flow allows gas to be delivered up to $8 \mathrm{~L} / \mathrm{min}$ in the neonate, promoting elimination of dead space and improvement in lung compliance. ${ }^{11,12}$ Benefits include ease of use, reduction in nasal trauma, lower pain scores, and improved nursing care and infant-parent bonding. ${ }^{13-15}$

\section{See the Related Editorial on Page 1428}

Several studies have shown HFNC to be a safe modality of providing respiratory support in premature neonates comparable with that of nasal CPAP. ${ }^{12,16-21}$ Extubation failure rates and work of breathing have been shown to be similar between the 2 modes of ventilation, even in the extremely premature. 12,18,21,22 Concerns continue to exist regarding the safety and efficacy of this modality, with the major criticism of the modality being the unpredictable generation of pulmonary distending pressures. ${ }^{11}$ However, current studies have not shown an increase in adverse outcomes with the use of HFNC over nasal CPAP. 12,16-21

The effect of the availability of multiple modes of noninvasive ventilator support in a single institution is currently unknown. Even with the implementation of weaning protocols, there is the potential for multimodality noninvasive respiratory support availability to have a profound effect on the respiratory support patterns of the premature infant. We aim to evaluate whether hospital stay and exposure to respiratory support was affected by the introduction of HFNC in addition to our existing respiratory support modalities.

\section{Methods}

\section{Study Design}

A retrospective chart review was conducted in a level 4 tertiary care neonatal ICU on subjects at 24-32 weeks' gestation requiring mid-level support, defined as noninvasive ventilatory support greater than low-flow nasal cannula during 2 periods surrounding HFNC implementation. This study was approved by the University of Maryland School of Medicine institutional review board. HFNC (Vapotherm, Vapotherm, Exeter, New Hampshire), was implemented in the neonatal ICU at the University of Maryland Medical Center in May of 2012. Before the introduction of HFNC, SiPAP (Carefusion, San Diego, California) and nasal CPAP (Carefusion, San Diego, California) were the modes of mid-level support available. The pre-HFNC period was from April 2011 to April 2012; the post-HFNC period was from August 2012 to August 2013. This al-

\section{QUICK LOOK}

\section{Current knowledge}

High-flow nasal cannula (HFNC) is a noninvasive respiratory support mode that is being increasingly used in neonates. Although the literature has not shown an increase in adverse outcomes, there continue to be concerns about the safety and efficacy of this modality.

\section{What this paper contributes to our knowledge}

This study evaluates the effect of HFNC introduction on respiratory support and disposition patterns. Our results, showing longer duration of respiratory support, decreased oral feeding, and decreased discharge to home after HFNC implementation, underline the need to closely examine new respiratory support modalities.

lowed 3 months for implementation of HFNC. For each period, demographic and clinical data were collected. The primary outcomes of the study were hospital stay and duration of respiratory support. Secondary outcomes included $\mathrm{BPD}$, defined as oxygen or positive pressure requirement at 36 weeks, gestation, home oxygen requirement, feeding pattern on discharge, and final disposition. Oxygen reduction tests were not used to establish the definition of BPD. In addition, we also evaluated outcomes of intraventricular hemorrhage, retinopathy of prematurity, patent ductus arteriosus, and necrotizing enterocolitis.

\section{Inclusion and Exclusion Criteria}

Infants were eligible for inclusion if they met the following criteria: (1) gestational age of 24 (0 of 7) to 32 (6 of 7) weeks and (2) required mid-level respiratory support, defined as use of HFNC, CPAP, or SiPAP. Patients were excluded for the following criteria: (1) death, (2) major congenital malformations, (3) age $>2$ weeks on admission, or (4) never weaned below mid-level respiratory support.

\section{Protocol Implementation}

Before HFNC implementation, education and protocol implementation were completed. Initiation, weaning, and failure guidelines were established. In the University of Maryland Medical Center neonatal ICU, HFNC was intended for use as an alternative to nasal CPAP for mild to moderate respiratory distress syndrome of any gestational age. Our institution used the flow cartridge with flow range of 2-6 L/min, with flow level being initiated at the discretion of the provider. Subjects were weaned at the discretion of the provider; however, general guidelines were 


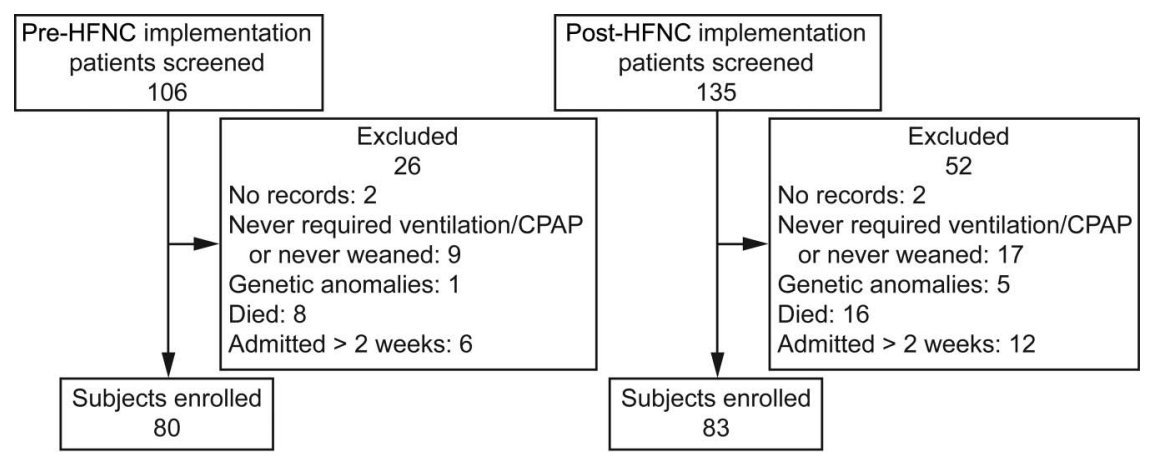

Fig. 1. Flow chart.

to wean by $1 \mathrm{~L} /$ min every $12 \mathrm{~h}$ if $\mathrm{F}_{\mathrm{IO}_{2}}$ requirement was $\leq 0.3$ with improved respiratory acidosis and work of breathing. HFNC should be discontinued once the patient is stable receiving $2 \mathrm{~L} / \mathrm{min}$. Per the guidelines, failure of HFNC should be considered for persistent apnea, $\mathrm{F}_{\mathrm{IO}_{2}}>0.6$, continued respiratory acidosis, or increased work of breathing despite maximizing HFNC support. Subjects were allowed to orally feed if support was $<3 \mathrm{~L} / \mathrm{min}$. All staff received in-service training on the device before implementation.

\section{Statistical Analysis}

All statistical analysis was performed using SAS 9.3 (SAS, Cary, North Carolina). Continuous variables are expressed as mean (SD), and categorical data are presented as $n(\%)$. Continuous data were analyzed using a $t$ test for parametric data and Wilcoxon rank-sum test for non-parametric data. Categorical data were analyzed using chi-square or Fisher exact testing. Multivariate linear and logistic regression was done to determine significant risk factors for primary outcomes controlling for covariates. Results for logistic regression are expressed as odds ratio and $95 \%$ CI. Statistical significance was set at $P<.05$.

\section{Results}

Of the 241 patients screened, 163 subjects were eligible, 80 in the pre-HFNC period and 83 in the post-HFNC period (Fig. 1). In the post-HFNC period, $68 \%$ of subjects were treated with HFNC, and of these subjects, $95 \%$ also were treated with nasal CPAP. In the post-HFNC period, the median number of HFNC failures (the requirement of nasal CPAP or intubation and ventilation) per subject was 0 with a range of $0-5$. The majority of subjects were exposed to nasal CPAP before starting HFNC, suggesting that in practice HFNC was mainly used as a weaning modality versus a nasal CPAP alternative.

Subjects were similar in their gestational age, birthweight, sex, race, 5-min Apgar score, delivery mode, and
Table 1. Study Period Baseline Characteristics

\begin{tabular}{lccc}
\hline \hline \multirow{2}{*}{\multicolumn{1}{c}{ Characteristics }} & \multicolumn{2}{c}{ Study Period } & \\
\cline { 2 - 3 } & $\begin{array}{c}\text { Pre-HFNC } \\
(n=80)\end{array}$ & $\begin{array}{c}\text { Post-HFNC } \\
(n=83)\end{array}$ & $P$ \\
\hline Gestational age, mean \pm SD y & $28.4 \pm 2.4$ & $28.2 \pm 2.1$ & .48 \\
Birthweight, mean \pm SD g & $1,125 \pm 420$ & $1,123 \pm 362$ & .97 \\
Male sex (\%) & $32(40)$ & $42(51)$ & .17 \\
5-min Apgar score, median (range) & $8(1-9)$ & $8(1-9)$ & .42 \\
Antenatal steroids, $n(\%)$ & $62(77.5)$ & $62(74.7)$ & .59 \\
& & & \\
\hline HFNC = high-flow nasal cannula & & & \\
\hline
\end{tabular}

Table 2. Study Period Outcomes

\begin{tabular}{lccc}
\hline \hline \multirow{2}{*}{ Outcomes } & \multicolumn{2}{c}{ Study Period } & \\
\cline { 2 - 3 } & $\begin{array}{c}\text { Pre-HFNC } \\
(n=80)\end{array}$ & $\begin{array}{c}\text { Post-HFNC } \\
(n=83)\end{array}$ & $P$ \\
\hline NEC, $n(\%)$ & $6(7.5)$ & $2(2.4)$ & .13 \\
ROP,$n(\%)$ & $20(25)$ & $35(43.2)$ & .02 \\
PDA, $n(\%)$ & $38(47.5)$ & $30(36)$ & .14 \\
IVH grade 3 or 4, $n(\%)$ & $9(11.4)$ & $6(7.2)$ & .36 \\
\hline HFNC $=$ high-flow nasal cannula & & & \\
NEC $=$ necrotizing enterocolitis & & & \\
ROP $=$ retinopathy of prematurity & & & \\
PDA $=$ patent ductus arteriousus & & & \\
IVH $=$ intraventricular hemorrhage & & & \\
& & & \\
\hline
\end{tabular}

antenatal steroid use (Table 1). There were no differences in the outcome of necrotizing enterocolitis, patent ductus arteriosus, culture-confirmed sepsis, and grade 3 and 4 intraventricular hemorrhage between the 2 groups (Table 2). However, in comparison with the pre-HFNC group, the post-HFNC group had higher rates of retinopathy of prematurity ( $25 \%$ vs $43.2 \%$, respectively, $P=.02$ ) (see Table 2 ). No difference was noted in the need for laser therapy between groups.

As compared with the pre-HFNC group, the duration of mid-level support was significantly longer in the post- 
Table 3. Respiratory Characteristics per Study Period

\begin{tabular}{|c|c|c|c|}
\hline \multirow[b]{2}{*}{ Characteristics } & \multicolumn{2}{|c|}{ Study Period } & \multirow[b]{2}{*}{$P$} \\
\hline & $\begin{array}{c}\text { Pre-HFNC } \\
(n=80)\end{array}$ & $\begin{array}{c}\text { Post-HFNC } \\
(n=83)\end{array}$ & \\
\hline $\mathrm{BPD}, n(\%)$ & $28(35)$ & $41(49.4)$ & .063 \\
\hline Ventilator days, mean \pm SD d & $8.3 \pm 14.5$ & $9.5 \pm 16.8$ & .62 \\
\hline Mid-level support days, mean \pm SD d & $15 \pm 15$ & $24 \pm 22$ & .004 \\
\hline Age reaching $\mathrm{LFNC}$, mean $\pm \mathrm{SD}$ d & $33 \pm 26$ & $52 \pm 41$ & .005 \\
\hline $\begin{array}{l}\text { HFNC }=\text { high-flow nasal cannula } \\
\text { BPD }=\text { bronchopulmonary dysplasia } \\
\text { LFNC = low-flow nasal cannula }\end{array}$ & & & \\
\hline
\end{tabular}

Table 4. Disposition per Study Period

\begin{tabular}{lccc}
\hline \hline \multicolumn{1}{c}{ Dispositions } & \multicolumn{2}{c}{ Study Period } & \\
\cline { 2 - 3 } & $\begin{array}{c}\text { Pre-HFNC } \\
(n=80)\end{array}$ & $\begin{array}{c}\text { Post-HFNC } \\
(n=83)\end{array}$ & $P$ \\
\hline CGA at discharge, mean \pm SD wks & $37 \pm 3.6$ & $37.3 \pm 4.9$ & .69 \\
LOS, mean \pm SD d & $60 \pm 33$ & $63 \pm 38$ & .53 \\
Per oral feeding on discharge, $n(\%)$ & $58(72.5)$ & $35(42.2)$ & $<.001$ \\
Discharge to home, $n(\%)$ & $54(67.5)$ & $36(43.4)$ & .002 \\
Discharge on oxygen, $n(\%)$ & $21(26.3)$ & $37(44.6)$ & .02 \\
& & & \\
\hline HFNC $=$ high-flow nasal cannula & & & \\
CGA $=$ corrected gestational age & & & \\
LOS $=$ length of stay & & & \\
\hline
\end{tabular}

HFNC group $(15 \pm 15 \mathrm{~d}$ vs $24 \pm 22 \mathrm{~d}$, respectively, $P=.004)$. The post-HFNC group was also older at the time of reaching a stable low-flow nasal cannula $(33 \pm 26 \mathrm{~d}$ vs $52 \pm 41 \mathrm{~d}$, pre- vs post-HFNC, $P=.005$ ). For those who were able to wean to room air before discharge, there was no difference between the 2 periods in the day of life during which they reached stable room air. There was no difference in ventilator days or failed extubation attempts between the 2 groups. Although the difference in BPD did not reach significance, there was a trend toward higher BPD rates in the post-HFNC group (35\% in the pre-HFNC group vs $49.4 \%$ in the post-HFNC group, $P=.063$ ) (Table 3 ).

The length of respiratory support and stay and corrected gestational age at the time of discharge were similar between the 2 groups (Table 4). However, those in the preHFNC period were more likely to be receiving full oral feeds at discharge $(72.5 \%$ vs $42.2 \%, P<.001)$. The preHFNC group was less likely to be discharged receiving oxygen $(26.3 \%$ vs $44.6 \%, P=.02)$. In the pre-HFNC period, $60.8 \%$ of subjects receiving oxygen at discharge were orally feeding versus only $20 \%$ in the post-HFNC group $(P=.001)$. The pre-HFNC group was also much more likely to be discharged home versus transferred to an intermediate care facility $(67.5 \%$ vs $43.4 \%, P=.002)$ (see Table 4$)$.
Adjusting for baseline demographic and clinical factors (birthweight, gestational age, sex, antenatal steroid use, 5-min Apgar score, patent ductus arteriosus, retinopathy of prematurity, necrotizing enterocolitis, ventilator days, and BPD status), study period remained a significant predictor of the length of time receiving mid-level respiratory support using multiple linear regression, with the post-HFNC period being associated with a 5.85-d increase in mid-level support (95\% CI $1.3-10.4$ d) $\left(\mathrm{r}^{2}=0.55, P=.01\right)$. Utilizing multiple logistic regression, study period also remained a significant predictor of oral feeding on discharge when controlling for these baseline demographic and clinical factors in addition to time receiving mid-level support (odds ratio $0.22,95 \%$ CI $0.097-0.508)(P<.001)$. When controlling for these baseline demographic and clinical factors as well as time receiving mid-level support, the only significant factor found for discharge to home versus to an intermediate care facility was oral feeding (odds ratio $<0.001,95 \%$ CI $<0.001-0.019)(P<.001)$.

When specifically evaluating the post-HFNC period, $67.5 \%$ were treated with HFNC. For subjects exposed to HFNC, the length of respiratory support and stay was significantly longer, 71.8 (42.2) versus 47.2 (19.8) days $(P<.001)$. There was also a trend toward decreased oral feeding on discharge for those subjects treated with multimodality support ( $35.9 \%$ vs $55.2 \%, P=.09$ ).

\section{Discussion}

In this retrospective study of infants $<33$ weeks' gestation requiring mid-level respiratory support, we evaluated respiratory patterns and patient outcomes before and after the introduction of HFNC. In the post-HFNC period, we saw a significant increase in time spent receiving midlevel respiratory support. In the post-HFNC period, those subjects who required low-flow nasal cannula were significantly older upon weaning to this modality. There was no difference in the age to room air between the 2 groups. In addition, we noted a trend in the post-HFNC period toward higher BPD rates, although this did not reach statistical significance. Interestingly, there were no differences in the gestational age, birthweight, number of ventilator days, or mode of invasive ventilation between the 2 periods. This underscores that the difference in noninvasive support between the 2 periods is less likely to be due to a major difference the severity of lung disease and more likely to be secondary to the respiratory support mode itself.

There is evidence to support that nasal CPAP promotes lung growth in the premature developing lung. The consistent positive pressure provided by nasal CPAP works to keep the alveoli open, maintain functional residual capacity, and reduce the work of breathing by stenting the airway and diaphragm..$^{23,24}$ In contrast, HFNC is a modality that works via washout of the nasopharyngeal dead space. 


\section{HFNC Use, Neonatal Respiratory Support Patterns and Length of Stay}

Unlike nasal CPAP, the HFNC prongs are designed not to occlude the nares, avoiding the creation of a seal for generating positive pressure. However, there have been several studies demonstrating that flows as low as $2 \mathrm{~L} / \mathrm{min}$ generate positive pressure as high as $6 \mathrm{~cm} \mathrm{H}_{2} \mathrm{O} .{ }^{25-28}$ To date, studies are inconsistent and show high variation in the pressures measured with HFNC. ${ }^{17,25-28}$ This difference in the mechanism of the modality may be a plausible explanation for the increased duration of respiratory support in this population. A randomized controlled trial by AbdelHady et al $^{29}$ showed that weaning subjects from nasal CPAP to nasal cannula was associated with an increased oxygen exposure and duration of respiratory support. This was echoed in a study done by Sasi et al, ${ }^{30}$ who also noted increased noninvasive respiratory times after initiation of HFNC. They felt that the inconsistent pressure generation could lead to progressive atelectasis, contributing to a prolonged duration receiving respiratory support. It may be that the consistent continuous positive pressure provided by modes such as nasal CPAP is more beneficial to premature lung development than the washout provided by HFNC.

Although we allowed a 3-month time frame for provider training and implementation, another explanation for prolonged noninvasive respiratory support in the post-HFNC period may be lack of provider comfort with the new modality and the availability of multiple modalities. This might lead to cycling through all available modalities and slower weaning. Although new technology has the potential to improve patient care, it is not without risks and requires careful consideration and implementation. ${ }^{31,32}$ Sant'Anna and Keszler ${ }^{33}$ advocate for a comprehensive respiratory support protocol to ensure optimal respiratory outcomes. They note that poor compliance after implementation of protocols is often due to lack of awareness or familiarity with the protocol. Although all of the providers in our institution received in-service training on both the device and the protocol, there may have continued to be some discomfort with the device that could lead to slow weaning. The time between training and actual use may have ranged beyond the 3-month period, depending on the provider's clinical schedule; this may have added to the lack of familiarity with the device. In addition, the ease of use of the device and increased patient tolerance may lead to slower weaning by the provider.

Although the increased respiratory support duration did not increase neonatal ICU stay, it may have contributed to a delay in discharge to home. Despite having a similar corrected gestational age at discharge, the post-HFNC period subjects were significantly less likely to be discharged home. In our institution, we discharge patients to a subacute facility to continue to work on oral feeding and weaning of respiratory support. An unexpected finding in this study was the delay to full oral feeding in the postHFNC period. Although our protocol allowed for oral feed- ing when support reached $<3 \mathrm{~L} / \mathrm{min}$, it may be that providers were hesitant to initiate oral feeds until patients were weaned to low-flow cannula. The majority of studies evaluating the use of HFNC did not include feeding pattern in their secondary outcomes. In the 2 studies we identified as examining feeding pattern differences as either a primary or secondary outcome, neither noted a difference in time to full oral feeds with the use of HFNC; however, neither study specified their feeding protocol for subjects receiving respiratory support. ${ }^{34,35}$ These findings are counterintuitive, since the HFNC setup should promote oral feeding. There is evidence that hypercapnia and subsequent increased respiratory drive may decrease the rate of sucking and swallowing in preterm infants. ${ }^{36}$ Although the modality itself should not directly interfere with oral feeding, the inconsistent pressure generation may lead to atelectasis and hypercapnia, which may indirectly delay feeding.

Another unexpected finding was the increased rate of retinopathy of prematurity. During the entire study time frame, we maintained the same targeted oxygen protocol. The only change during the post-HFNC period as compared with the pre-HFNC period was a change in ophthalmologists and a use of camera technology that may have led to increased diagnosis of the lower stages. This idea is supported by the fact that there was no difference in the need for laser therapy between groups. Although this 1-y pre- and post-intervention study is limited by its retrospective design and relatively small numbers, we were able to show strong trends in respiratory support use and disposition status between the 2 periods. The similarity in baseline characteristics in the 2 groups is a clear strength of the study. Also, limiting the overall study time frame to $2 \mathrm{y}$ decreases confounding factors of practice variability that may affect patient outcomes.

\section{Conclusions}

The popularity of HFNC is increasing secondary to its ease of use and increased patient tolerance. However, in our institution, the introduction to HFNC was associated with significantly increased time receiving respiratory support, decreased oral feeding on discharge, and decreased rates of discharge to home. This study underlines the need to closely examine new respiratory support modalities on an ongoing basis to ensure optimal respiratory outcomes.

\section{REFERENCES}

1. McCabe ER, Carrino GE, Russell RB, Howse JL. Fighting for the next generation: US prematurity in 2030. Pediatrics 2014;134(6): 1193-1199.

2. Angus DC, Linde-Zwirble WT, Clermont G, Griffin MF, Clark RH. Epidemiology of neonatal respiratory failure in the United States: projections from California and New York. Am J Respir Crit Care Med 2001;164(7):1154-1160. 


\section{HFNC Use, Neonatal Respiratory Support Patterns and Length of Stay}

3. Davis PG, Henderson-Smart DJ. Nasal continuous positive airways pressure immediately after extubation for preventing morbidity in preterm infants. Cochrane Database Syst Rev 2003;(2):CD000143.

4. Subramaniam P, Henderson-Smart DJ, Davis PG. Prophylactic nasal continuous positive airways pressure for preventing morbidity and mortality in very preterm infants. Cochrane Database Syst Rev 2005; (3):CD001243.

5. Morley CJ, Davis PG, Doyle LW, Brion LP, Hascoet JM, Carlin JB, COIN Trial Investigators. Nasal CPAP or intubation at birth for very preterm infants. N Engl J Med 2008;358(7):700-708.

6. Aly $\mathrm{H}$, Herson V. Nasal continuous positive airway pressure and Gram-negative sepsis in low-birthweight infants. Pediatr Infect Dis J 2006;25(7):663-664; author reply 664.

7. Bonner KM, Mainous RO. The nursing care of the infant receiving bubble CPAP therapy. Adv Neonatal Care 2008;8(2):78-95.

8. McCoskey L. Nursing Care Guidelines for prevention of nasal breakdown in neonates receiving nasal CPAP. Adv Neonatal Care 2008; 8(2):116-124.

9. Ojha S, Gridley E, Dorling J. Use of heated humidified high-flow nasal cannula oxygen in neonates: a UK wide survey. Acta Paediatr 2013;102(3):249-253.

10. Manley BJ, Owen L, Doyle LW, Davis PG. High-flow nasal cannulae and nasal continuous positive airway pressure use in non-tertiary special care nurseries in Australia and New Zealand. J Paediatr Child Health 2012;48(1):16-21.

11. Manley BJ, Dold SK, Davis PG, Roehr CC. High-flow nasal cannulae for respiratory support of preterm infants: a review of the evidence. Neonatology 2012;102(4):300-308.

12. Saslow JG, Aghai ZH, Nakhla TA, Hart JJ, Lawrysh R, Stahl GE, Pyon KH. Work of breathing using high-flow nasal cannula in preterm infants. J Perinatol 2006;26(8):476-480.

13. Hough JL, Shearman AD, Jardine LA, Davies MW. Humidified high flow nasal cannulae: current practice in Australasian nurseries, a survey. J Paediatr Child Health 2012;48(2):106-113.

14. Osman M, Elsharkawy A, Abdel-Hady H. Assessment of pain during application of nasal-continuous positive airway pressure and heated, humidified high-flow nasal cannulae in preterm infants. J Perinatol 2015;35(4):263-267.

15. Armfield M, West G. Use of Vapotherm for respiratory support with neonates. Paediatr Nurs 2009;21(1):27-30.

16. Holleman-Duray D, Kaupie D, Weiss MG. Heated humidified highflow nasal cannula: use and a neonatal early extubation protocol. J Perinatol 2007;27(12):776-781.

17. Collins CL, Holberton JR, König K. Comparison of the pharyngeal pressure provided by two heated, humidified high-flow nasal cannulae devices in premature infants. J Paediatr Child Health 2013; 49(7):554-556.

18. Collins CL, Holberton JR, Barfield C, Davis PG. A randomized controlled trial to compare heated humidified high-flow nasal cannulae with nasal continuous positive airway pressure postextubation in premature infants. J Pediatr 2013;162(5):949-954.e1.

19. Collins CL, Barfield C, Horne RS, Davis PG. A comparison of nasal trauma in preterm infants extubated to either heated humidified highflow nasal cannulae or nasal continuous positive airway pressure. Eur J Pediatr 2014;173(2):181-186.
20. Campbell DM, Shah PS, Shah V, Kelly EN. Nasal continuous positive airway pressure from high flow cannula versus Infant Flow for Preterm infants. J Perinatol 2006;26(9):546-549.

21. Fernandez-Alvarez JR, Gandhi RS, Amess P, Mahoney L, Watkins $R$, Rabe H. Heated humidified high-flow nasal cannula versus lowflow nasal cannula as weaning mode from nasal CPAP in infants $\leq 28$ weeks of gestation. Eur J Pediatr 2014;173(1):93-98.

22. Manley BJ, Owen LS, Doyle LW, Andersen CC, Cartwright DW, Pritchard MA, et al. High-flow nasal cannulae in very preterm infants after extubation. N Engl J Med 2013;369(15):1425-1433.

23. Aly H, Milner JD, Patel K, El-Mohandes AA. Does the experience with the use of nasal continuous positive airway pressure improve over time in extremely low birth weight infants? Pediatrics 2004; 114(3):697-702.

24. Diblasi RM. Nasal continuous positive airway pressure (CPAP) for the respiratory care of the newborn infant. Respir Care 2009;54(9): 1209-1235.

25. Wilkinson DJ, Andersen CC, Smith K, Holberton J. Pharyngeal pressure with high-flow nasal cannulae in premature infants. J Perinatol 2008;28(1):42-47.

26. Hasan RA, Habib RH. Effects of flow rate and airleak at the nares and mouth opening on positive distending pressure delivery using commercially available high-flow nasal cannula systems: a lung model study. Pediatr Crit Care Med 2011;12(1):e29-e33.

27. Locke RG, Wolfson MR, Shaffer TH, Rubenstein SD, Greenspan JS. Inadvertent administration of positive end-distending pressure during nasal cannula flow. Pediatrics 1993;91(1):135-138.

28. Sreenan C, Lemke RP, Hudson-Mason A, Osiovich H. High-flow nasal cannulae in the management of apnea of prematurity: a comparison with conventional nasal continuous positive airway pressure. Pediatrics 2001;107(5):1081-1083.

29. Abdel-Hady H, Shouman B, Aly H. Early weaning from CPAP to high flow nasal cannula in preterm infants is associated with prolonged oxygen requirement: a randomized controlled trial. Early Hum Dev 2011;87(3):205-208.

30. Sasi A, Malhotra A. High flow nasal cannula for continuous positive airway pressure weaning in preterm neonates: a single-centre experience. J Paediatr Child Health 2015;51(2):199-203.

31. Poulin P, Austen L, Scott CM, Poulin M, Gall N, Seidel J, et al. Introduction of new technologies and decision making processes: a framework to adapt a Local Health Technology Decision Support Program for other local settings. Med Devices (Auckl) 2013;6:185-193.

32. Aggarwal R, Mytton OT, Greaves F, Vincent C. Technology as applied to patient safety: an overview. Qual Saf Health Care 2010; 19(Suppl 2):i3-i8.

33. Sant'Anna GM, Keszler M. Developing a neonatal unit ventilation protocol for the preterm baby. Early Hum Dev 2012;88(12):925-929.

34. Amendolia B, Fisher K, Wittmann-Price RA, Bloch JR, Gardner M, Basit M, Aghai ZH. Feeding tolerance in preterm infants on noninvasive respiratory support. J Perinat Neonatal Nurs 2014;28(4):300-304.

35. Yoder BA, Stoddard RA, Li M, King J, Dirnberger DR, Abbasi S. Heated, humidified high-flow nasal cannula versus nasal CPAP for respiratory support in neonates. Pediatrics 2013;131(5):e1482-e1490.

36. Timms BJ, DiFiore JM, Martin RJ, Miller MJ. Increased respiratory drive as an inhibitor of oral feeding of preterm infants. J Pediatr 1993; 123(1):127-131.

This article is approved for Continuing Respiratory Care Education credit. For information and to obtain your $\mathrm{CRCE}$

(free to AARC members) visit www.rcjournal.com

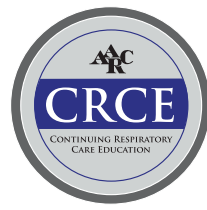

